\title{
Galerkin Methods for Singular Boundary Value Problems in One Space Dimension
}

\author{
By Kenneth Eriksson and Vidar Thomée
}

\begin{abstract}
Two Galerkin type piecewise polynomial approximation procedures based on bilinear forms with different weight functions are analyzed and compared. Optimal order error estimates are proved and numerical results are presented.
\end{abstract}

1. Introduction. In this paper we shall discuss Galerkin piecewise polynomial approximation methods for the singular two-point boundary value problem

$$
\begin{gathered}
L u(x) \equiv-u^{\prime \prime}(x)-\frac{b}{x} u^{\prime}(x)+q(x) u(x)=f(x), \quad x \in I \equiv(0,1), \\
u^{\prime}(0)=u(1)=0,
\end{gathered}
$$

and also for the corresponding time-dependent problem

$$
\begin{gathered}
u_{t}(x, t)+L u(x, t)=f(x, t), \quad x \in I, t>0, \\
u^{\prime}(0, t)=u(1, t)=0, \quad t>0, \\
u(x, 0)=v(x), \quad x \in I,
\end{gathered}
$$

where $u_{t}=\partial u / \partial t$ and $u^{\prime}=\partial u / \partial x$, and where $b$ is a positive constant, $q=q(x)$ is a bounded nonnegative function, and $f$ and $v$ are given data. We shall always assume that these problems admit unique solutions which are sufficiently smooth for our purposes. Note that if $u \in C^{2}(\bar{I})$ and if $f$ is bounded at zero, then the boundary condition there is automatically satisfied. In fact, for $b \geqslant 1$ it is easy to see that if $u \in C^{2}((0,1])$ and $u$ and $f$ are bounded at zero, then $u \in C^{1}(\bar{I})$ and $u^{\prime}(0)=0$.

Problems of the form (1.1) and (1.2) arise naturally from spherically symmetric problems in higher dimensions. For example, if $u=u(\mathbf{x})$ with $\mathbf{x}=\left(x_{1}, \ldots, x_{n}\right)$ is the solution of the Dirichlet problem

$$
\begin{aligned}
-\Delta u+q u=f & \text { in } B, \\
u=0 & \text { on } \partial B,
\end{aligned}
$$

where $B=B_{1}(0)$ is the unit ball in $R^{n}$ and $\Delta$ the Laplacian, and where $q$ and $f$ depend only on $|\mathbf{x}|$, then $u$ depends only on $|\mathbf{x}|$ and, introducing polar coordinates with $x=|\mathbf{x}|$, one finds that $u=u(x)$ is the solution of (1.1) with $b=n-1$.

Received June 30, 1982.

1980 Mathematics Subject Classification. Primary 65N15, 65N30.

Key words and phrases. Singular two-point boundary value problem, singular parabolic equation, spherical symmetry, piecewise polynomial approximation, Galerkin's method, error estimate. 
Also, the problem of finding a bounded solution of

$$
\begin{aligned}
-v^{\prime \prime}+Q(y) v & =F(y), \quad y \in(1, \infty), \\
v(1) & =0,
\end{aligned}
$$

reduces to (1.1) by means of the transformation of variable $y=x^{-\alpha}$ for $\alpha>0$, giving $b=1+\alpha, q(x)=\alpha^{2} Q\left(x^{-\alpha}\right) x^{-2-2 \alpha}$, and $f(x)=\alpha^{2} F\left(x^{-\alpha}\right) x^{-2-2 \alpha}$.

We shall consider approximate solutions of (1.1) and (1.2) in the finite dimensional space $S_{h}$ of continuous functions which vanish at $x=1$ and which reduce to polynomials of degree at most $r-1$ on each subinterval $I_{i}=\left(x_{i-1}, x_{i}\right)$ of the partition of $I$ defined by $0=x_{0}<x_{1}<\cdots<x_{N}=1$. We set $h_{i}=x_{i}-x_{i-1}$, $h=\max _{1 \leqslant i \leqslant N} h_{i}$, and $\beta=h^{-1} \min _{i} h_{i}$, where the latter quantity measures the uniformity of the partition. Throughout the paper we denote by $C$ various constants which may depend on $b, q, \beta$, and $r$, but not on $h$ and the data of (1.1) and (1.2).

We first consider the stationary problem (1.1). Writing the differential equation in the form

$$
-\left(x^{b} u^{\prime}\right)^{\prime}+x^{b} q u=x^{b} f
$$

we find at once that $u$ also solves the variational problem

$$
A(u, \varphi)=\left(x^{b} f, \varphi\right) \quad \forall \varphi \in \dot{H}^{1},
$$

where $A(\cdot, \cdot)$ denotes the symmetric bilinear form

$$
A(u, \varphi)=\int_{0}^{1}\left(x^{b} u^{\prime} \varphi^{\prime}+x^{b} q u \varphi\right) d x,
$$

where $(\cdot, \cdot)$ is the inner product in $L_{2}(I)$, and where $\dot{H}^{1}$ is the space of all $v \in$ $C((0,1])$ which vanish at $x=1$ and for which $x^{b / 2} v^{\prime} \in L_{2}$. We are therefore led to pose as a discrete analogue of (1.1) the problem of finding $u_{h} \in S_{h}$ such that

$$
A\left(u_{h}, \chi\right)=\left(x^{b} f, \chi\right) \quad \forall \chi \in S_{h} .
$$

Using straightforward variational methods, Eisenstat, Schreiber and Schultz [3], [8] have shown the following weighted norm error estimate for this approximation, namely

$$
\left\|x^{b / 2}\left(u_{h}-u\right)\right\| \leqslant C h^{r}\left\|x^{b / 2} u^{(r)}\right\|,
$$

where $\|\cdot\|$ denotes the norm in $L_{2}(I)$ and $u^{(r)}$ the $r$ th derivative of $u$, and, by a somewhat more refined analysis, Jespersen [6] was able to derive the uniform error estimate

$$
\left\|u_{h}-u\right\|_{L_{x}} \leqslant C\left(\ln \frac{1}{h}\right)^{\bar{r}} h^{r}\left\|u^{(r)}\right\|_{L_{x}}
$$

where $\bar{r}=1$ if $r=2$ and $\bar{r}=0$ if $r>2$. For completeness and ease of reference we shall demonstrate (1.4) in Section 2 below.

Turning to the time-dependent problem (1.2), we take the analogous approach and propose in Section 3, as a first step towards a complete discretization, the semidiscrete problem to find $u_{h}=u_{h}(t) \in S_{h}$ such that

$$
\begin{gathered}
\left(x^{b} u_{h, t}, \chi\right)+A\left(u_{h}, \chi\right)=\left(x^{b} f, \chi\right) \quad \forall \chi \in S_{h}, t>0, \\
u_{h}(0)=v_{h},
\end{gathered}
$$


where $v_{h} \in S_{h}$ is a suitable approximation of $v$. Given a basis for $S_{h}$ this variational problem reduces to a nonsingular linear system of ordinary differential equations and therefore admits a unique solution. For the error in the semidiscrete approximation we prove the estimate

$$
\begin{aligned}
\left\|x^{b / 2}\left(u_{h}(t)-u(t)\right)\right\| \leqslant & \left\|x^{b / 2}\left(v_{h}-v\right)\right\| \\
& +C h^{r}\left\{\left\|x^{b / 2} v^{(r)}\right\|+\int_{0}^{t}\left\|x^{b / 2} u_{t}^{(r)}\right\| d s\right\} .
\end{aligned}
$$

Having once obtained (1.4), the proof of (1.6) essentially reduces to a comparison of $u_{h}$ and the projection $\tilde{u}_{h} \in S_{h}$ of $u$ defined by

$$
A\left(\tilde{u}_{h}-u, \chi\right)=0 \quad \forall \chi \in S_{h} .
$$

In fact, it can be shown that

$$
\left\|x^{b / 2}\left(\tilde{u}_{h}^{\prime}-u_{h}^{\prime}\right)\right\|=O\left(h^{r}\right)
$$

which is one power of $h$ better than one might first expect. This type of superconvergence was used by Wheeler [9] to derive optimal order uniform convergence for the approximate solution of a nonsingular problem. Using similar arguments we obtain essentially optimal order uniform convergence for $x$ bounded away from zero, or, more precisely, for a suitable choice of $v_{h}$ and for $\alpha \in(0,1)$,

$$
\left\|u_{h}(t)-u(t)\right\|_{L_{x}(\alpha, 1)} \leqslant C_{\alpha} h^{r}\left\{\left(\ln \frac{1}{h}\right)^{r}\left\|u^{(r)}(t)\right\|_{L_{\infty}}+\left(\int_{0}^{t}\left\|x^{h / 2} u_{t}^{(r)}\right\|^{2} d s\right)^{1 / 2}\right\} .
$$

For $0 \leqslant b<1$ one may, in fact, take $\alpha=0$.

However, numerical experiments using the above procedures for both the stationary and the time-dependent problems show a marked loss of accuracy near $x=0$. Since this appears to be caused by the weight $x^{b}$ in the weak formulation of the problem, we shall consider an alternative approach for $b>1$.

We return first to the stationary equation (1.1), which we now write in the form

$$
-\left(x u^{\prime}\right)^{\prime}-(b-1) u^{\prime}+x q u=x f,
$$

and observe that $u$ satisfies the variational equation

$$
B(u, \chi)=(x f, \chi) \quad \forall \chi \in S_{h},
$$

where $B(\cdot, \cdot)$ is the nonsymmetric bilinear form

$$
B(u, \chi)=\left(x u^{\prime}, \chi^{\prime}\right)-(b-1)\left(u^{\prime}, \chi\right)+(x q u, \chi) .
$$

For $b>1$ we therefore propose the discrete problem to find $u_{h} \in S_{h}$ such that

$$
B\left(u_{h}, \chi\right)=(x f, \chi) \quad \forall \chi \in S_{h} .
$$

Note that for $b=1$ this method coincides with the previous symmetric one. Note also that $B(\cdot, \cdot)$ is positive and that hence (1.8) admits a unique solution. In fact, for $v \not \equiv 0$ vanishing at $x=1$ we have

$$
B(v, v)=\left(x v^{\prime}, v^{\prime}\right)+\frac{b-1}{2} v^{2}(0)+(x q v, v)>0 .
$$

A natural norm associated with the above nonsymmetric variational formulation of the problem and therefore suitable for error estimates would be $\left\|x^{1 / 2} \cdot\right\|$. Instead 
of pursuing an analysis in this norm we shall concentrate on the uniform norm and devote Section 4 to showing the optimal order estimate

$$
\left\|u_{h}-u\right\|_{L_{\infty}} \leqslant C h^{r}\left\|u^{(r)}\right\|_{L_{\infty}} .
$$

Turning now to the time-dependent problem, again with $b>1$, we shall study in Section 5 the semidiscrete approximate solution $u_{h}=u_{h}(t) \in S_{h}$ defined by

$$
\begin{gathered}
\left(x u_{h, t}, \chi\right)+B\left(u_{h}, \chi\right)=(x f, \chi) \quad \forall \chi \in S_{h}, t>0, \\
u_{h}(0)=v_{h} .
\end{gathered}
$$

We first show that if $v_{h}$ is chosen as the elliptic projection $P_{B} v \in S_{h}$ of $v$ defined by

$$
B\left(P_{B} v-v, \chi\right)=0 \quad \forall \chi \in S_{h},
$$

then

$$
\left\|u_{h}(t)-u(t)\right\|_{L_{\infty}} \leqslant C h^{r}\left(\ln \frac{1}{h}\right)^{1 / 2}\left\{\left\|u^{(r)}(t)\right\|_{L_{\infty}}+\left\|u_{t}^{(r)}(0)\right\|_{L_{\infty}}+\int_{0}^{t}\left\|u_{t t}^{(r)}\right\|_{L_{\infty}} d s\right\} .
$$

We then use a smoothing property of the solution operator of the homogeneous semidiscrete equation to remove the restrictions on $v_{h}$, and show

$$
\begin{aligned}
\left\|u_{h}(t)-u(t)\right\|_{L_{\infty}} \leqslant & C\left(\ln \frac{1}{h}\right) t^{-1 / 2}\left\|x^{1 / 2}\left(P_{B} v-v_{h}\right)\right\| \\
& +C h^{r}\left(\ln \frac{1}{h}\right)^{1 / 2}\left\{\left\|u^{(r)}(t)\right\|_{L_{\infty}}+\left\|u_{t}^{(r)}(0)\right\|_{L_{\infty}}+\int_{0}^{t}\left\|u_{t t}^{(r)}\right\|_{L_{\infty}} d s\right\} .
\end{aligned}
$$

In practice our above methods require the evaluation by numerical quadrature of the coefficients involving $q$ and $f$. As an example we demonstrate in Section 6, for the nonsymmetric stationary problem, how quadrature rules may be chosen so as to retain the convergence properties of the exact semidiscrete solution.

Finally, in Section 7 we show some numerical results from computations on some test problems.

For other treatments of problems of the type considered here, see, for instance ,[1], [2], [4], [5], and [7], and references in these.

In addition to the usual Banach spaces $L_{p}=L_{p}(I)$ with norms $\|\cdot\|_{L_{p}}(\|\cdot\|$ when $p=2$ ), and the Sobolev spaces $W_{p}^{k}$ with norms

$$
\|v\|_{W_{p}^{k}}=\left(\sum_{j \leqslant k}\left\|v^{(j)}\right\|_{L_{p}}^{p}\right)^{1 / p}
$$

we shall use the piecewise Sobolev norms defined by

$$
\|v\|_{W_{p}^{k, h}}=\left(\sum_{i=1}^{N}\|v\|_{W_{p}^{k}\left(I_{i}\right)}^{p}\right)^{1 / p}
$$

If the domain considered is a subinterval of $I$, this is specified in the notation. Also, we denote by $\|A\|_{l_{\infty}}$ the matrix norm $\max _{i} \Sigma_{j}\left|a_{i j}\right|$, where $A=\left(a_{i j}\right)$.

2. The Symmetric Method. In this section we shall consider the symmetric variational problem to find $u \in \dot{H}^{1}$ such that

$$
A(u, \varphi)=\left(x^{b} u^{\prime}, \varphi^{\prime}\right)+\left(x^{b} q u, \varphi\right)=\left(x^{b} f, \varphi\right) \quad \forall \varphi \in \dot{H}^{1},
$$


and its discrete analogue to find $u_{h} \in S_{h}$ with

$$
A\left(u_{h}, \chi\right)=\left(x^{b} f, \chi\right) \quad \forall \chi \in S_{h} .
$$

Note that since $S_{h} \subset \dot{H}^{1}$, it follows from (2.1) and (2.2) that the error $u_{h}-u$ is orthogonal to $S_{h}$ with respect to the bilinear form $A(\cdot, \cdot)$, or

$$
A\left(u_{h}-u, \chi\right)=0 \quad \forall \chi \in S_{h} .
$$

This also defines $u_{h}$ as a projection of $u$ onto $S_{h}$.

Assuming that the solution of (2.1) is sufficiently smooth, we shall prove the following error estimate.

THEOREM 1. Let $u$ and $u_{h}$ be the solutions of (2.1) and (2.2), respectively. Then

$$
\left\|x^{b / 2}\left(u_{h}-u\right)\right\| \leqslant C h^{r}\left\|x^{b / 2} u^{(r)}\right\| .
$$

Before we proceed to prove this result we state a simple Poincaré-type inequality and also an approximation property of $S_{h}$.

LEMMA 1. For $\alpha \geqslant 0,1 \leqslant p \leqslant \infty, d>0$, and functions $v$ with $v(d)=0$, we have

$$
\left\|x^{\alpha} v\right\|_{L_{p}(0, d)} \leqslant d\left\|x^{\alpha} v^{\prime}\right\|_{L_{p}(0, d)} .
$$

Proof. We have at once

$$
\left|x^{\alpha} v(x)\right|=\left|x^{\alpha} \int_{x}^{d} s^{-\alpha} s^{\alpha} v^{\prime}(s) d s\right| \leqslant\left\|x^{\alpha} v^{\prime}\right\|_{L_{1}(0, d)},
$$

so that by Hölder's inequality

$$
\begin{aligned}
\left\|x^{\alpha} v\right\|_{L_{p}(0, d)} & \leqslant d^{1 / p}\left\|x^{\alpha} v\right\|_{L_{\infty}(0, d)} \leqslant d^{1 / p}\left\|x^{\alpha} v^{\prime}\right\|_{L_{1}(0, d)} \\
& \leqslant d\left\|x^{\alpha} v^{\prime}\right\|_{L_{p}(0, d)},
\end{aligned}
$$

which is the desired estimate.

LEMMA 2. There is a constant $C=C(b, \beta)$ and for each integer $m$ with $1 \leqslant m \leqslant r$ and each appropriately regular $v$ with $v(1)=0$ an interpolant $\bar{v} \in S_{h}$ of $v$ such that

$$
\left\|x^{b / 2}\left(v^{\prime}-\bar{v}^{\prime}\right)\right\| \leqslant C h^{m-1}\left\|x^{b / 2} v^{(m)}\right\| .
$$

Proof. On the first interval $I_{1}$, let $\bar{v}$ be the polynomial of degree $m-1$ determined by

$$
\vec{v}^{(j)}\left(x_{1}\right)=v^{(j)}\left(x_{1}\right), \quad j=0, \ldots, m-1 .
$$

On the remaining intervals, let $\bar{v} \in P_{m-1}\left(I_{i}\right)$ interpolate $v$ at any $m$ distinct points including the endpoints. It is well known that for such an interpolant

$$
\left\|v^{\prime}-\bar{v}^{\prime}\right\|_{L_{2}\left(I_{i}\right)} \leqslant h_{i}^{m-1}\left\|v^{(m)}\right\|_{L_{2}\left(I_{i}\right)}
$$

so that for $i>1$

$$
\begin{aligned}
\left\|x^{b / 2}\left(v^{\prime}-\bar{v}^{\prime}\right)\right\|_{L_{2}\left(I_{i}\right)}^{2} & \leqslant x_{i}^{b} x_{i-1}^{-b} h_{i}^{2 m-2}\left\|x^{b / 2} v^{(m)}\right\|_{L_{2}\left(I_{i}\right)}^{2} \\
& \leqslant C(b, \beta) h^{2 m-2}\left\|x^{b / 2} v^{(m)}\right\|_{L_{2}\left(I_{i}\right)}^{2} .
\end{aligned}
$$

Repeated use of Lemma 1 shows the analogous estimate for $i=1$. Summation over $i$ then yields the desired estimate and completes the proof. 
We are now ready to give the

Proof of Theorem 1. With $e=u_{h}-u$ we shall first prove directly from the variational formulation that

$$
\left\|x^{b / 2} e^{\prime}\right\| \leqslant C h^{r-1}\left\|x^{b / 2} u^{(r)}\right\|
$$

and then, by a duality argument, that

$$
\left\|x^{b / 2} e\right\| \leqslant C h\left\|x^{b / 2} e^{\prime}\right\| .
$$

Together these estimates prove Theorem 1.

By our assumptions on $q$ and by (2.3) we have

$$
\begin{aligned}
\left\|x^{b / 2} e^{\prime}\right\|^{2} & \leqslant A(e, e)=A(e, u-\chi) \\
& \leqslant C(q)\left\|x^{b / 2} e^{\prime}\right\|\left\|x^{b / 2}\left(u^{\prime}-\chi^{\prime}\right)\right\| \quad \forall \chi \in S_{h},
\end{aligned}
$$

where we have used Lemma 1 in the last step. In view of Lemma 2 this proves (2.4).

In preparation for the proof of $(2.5)$, note that the space $\dot{H}^{1}$ defined in the introduction may be equipped with the inner product $A(\cdot, \cdot)$ and the corresponding norm to form a Hilbert space. By the Riesz representation theorem there is a unique solution $\phi \in \dot{H}^{1}$ of (the adjoint problem)

$$
A(\varphi, \phi)=\left(x^{b} \varphi, e\right) \quad \forall \varphi \in \dot{H}^{1} .
$$

Setting $\varphi=e$ and using (2.3) and Lemma 2, we have for a suitable $\chi$

$$
\begin{aligned}
\left\|x^{b / 2} e\right\|^{2} & =A(e, \phi)=A(e, \phi-\chi) \\
& \leqslant C\left\|x^{b / 2} e^{\prime}\right\|\left\|x^{b / 2}\left(\phi^{\prime}-\chi^{\prime}\right)\right\| \\
& \leqslant C h\left\|x^{b / 2} e^{\prime}\right\|\left\|x^{b / 2} \phi^{\prime \prime}\right\| .
\end{aligned}
$$

It remains then to show that, for some constant $C=C(b, q)$,

$$
\left\|x^{b / 2} \phi^{\prime \prime}\right\| \leqslant C\left\|x^{b / 2} e\right\| .
$$

For this purpose we note that the problem corresponding to $q=0$,

$$
\left(x^{b} \varphi^{\prime}, \psi^{\prime}\right)=\left(x^{b} \varphi, g\right) \quad \forall \varphi \in \dot{H}^{1},
$$

has the unique solution

$$
\psi(x)=\int_{x}^{1} y^{-b} \int_{0}^{b} s^{b} g(s) d s,
$$

and differentiating twice we obtain

$$
\psi^{\prime \prime}(x)=-g(x)+b x^{-b-1} \int_{0}^{x} s^{b} g(s) d s .
$$

Recalling Hardy's inequality,

$$
\left\|x^{-1} \int_{0}^{x} f d s\right\| \leqslant 2\|f\|
$$

we find

$$
\left\|x^{b / 2} \psi^{\prime \prime}\right\| \leqslant\left\|x^{b / 2} g\right\|+b\left\|x^{-1} \int_{0}^{x}(s / x)^{b / 2} s^{b / 2} g d s\right\| \leqslant C\left\|x^{b / 2} g\right\| .
$$

Since $\phi$ solves the equation (2.8) with $g=e-q \phi$, we conclude

$$
\left\|x^{b / 2} \phi^{\prime \prime}\right\| \leqslant C\left(\left\|x^{b / 2} e\right\|+\left\|x^{b / 2} \phi\right\|\right) .
$$


On the other hand, we have at once from Lemma 1 and (2.6) that

$$
\left\|x^{b / 2} \phi\right\|^{2} \leqslant A(\phi, \phi)=\left(x^{b} \phi, e\right) \leqslant\left\|x^{b / 2} \phi\right\|\left\|x^{b / 2} e\right\|
$$

so that

$$
\left\|x^{b / 2} \phi\right\| \leqslant\left\|x^{b / 2} e\right\| .
$$

Together (2.9) and (2.10) complete the proof of (2.7) and hence of the theorem.

3. The Symmetric Method for the Time-Dependent Problem. In this section we shall consider the semidiscrete parabolic problem

$$
\begin{gathered}
\left(x^{b} u_{h, t}, \chi\right)+A\left(u_{h}, \chi\right)=\left(x^{b} f, \chi\right) \quad \forall \chi \in S_{h}, t>0, \\
u_{h}(0)=v_{h},
\end{gathered}
$$

where again $A(\cdot, \cdot)$ is the symmetric bilinear form defined in (1.3). We shall first show the following weighted norm estimate.

THEOREM 2. Let $u$ and $u_{h}$ be the solutions of (1.2) and (3.1), respectively. Then

$$
\begin{aligned}
\left\|x^{b / 2}\left(u_{h}(t)-u(t)\right)\right\| \leqslant & \left\|x^{b / 2}\left(v_{h}-v\right)\right\| \\
& +C h^{r}\left\{\left\|x^{b / 2} v^{(r)}\right\|+\int_{0}^{t}\left\|x^{b / 2} u_{t}^{(r)}\right\| d s\right\} .
\end{aligned}
$$

Proof. We define the elliptic projection $P_{A}$ onto $S_{h}$ by

$$
A\left(P_{A} u-u, \chi\right)=0 \quad \forall \chi \in S_{h}
$$

and write

$$
u_{h}-u=\left(u_{h}-P_{A} u\right)+\left(P_{A} u-u\right)=\theta+\rho .
$$

We first note that, by the error estimate of Theorem 1 ,

$$
\begin{aligned}
\left\|x^{b / 2} \rho(t)\right\| & \leqslant C h^{r}\left\|x^{b / 2} u^{(r)}(t)\right\| \\
& \leqslant C h^{r}\left\{\left\|x^{b / 2} v^{(r)}\right\|+\int_{0}^{t}\left\|x^{b / 2} u_{t}^{(r)}\right\| d s\right\} .
\end{aligned}
$$

Combining (3.1) and the corresponding weak form of (1.2) with (3.2), we find for $\theta \in S_{h}$ that

$$
\left(x^{b} \theta_{t}, \chi\right)+A(\theta, \chi)=-\left(x^{b} \rho_{t}, \chi\right) \quad \forall \chi \in S_{h} .
$$

With $\chi=\theta$ this yields

$$
\frac{1}{2} \frac{d}{d t}\left\|x^{b / 2} \theta\right\|^{2}+A(\theta, \theta)=-\left(x^{b} \rho_{t}, \theta\right) \leqslant\left\|x^{b / 2} \rho_{t}\right\|\left\|x^{b / 2} \theta\right\|,
$$

from which we conclude

$$
\frac{d}{d t}\left\|x^{b / 2} \theta\right\| \leqslant\left\|x^{b / 2} \rho_{t}\right\|
$$

and hence, using now Theorem 1 also to estimate $\rho_{t}$,

$$
\begin{aligned}
\left\|x^{b / 2} \theta(t)\right\| & \leqslant\left\|x^{b / 2}\left(v_{h}-P_{A} v\right)\right\|+\int_{0}^{t}\left\|x^{b / 2} \rho_{t}\right\| d s \\
& \leqslant\left\|x^{b / 2}\left(v_{h}-v\right)\right\|+C h^{r}\left\{\left\|x^{b / 2} v^{(r)}\right\|+\int_{0}^{t}\left\|x^{b / 2} u_{t}^{(r)}\right\| d s\right\} .
\end{aligned}
$$

Together (3.3) and (3.5) prove the theorem. 
We shall show now that a similar argument yields a superconvergent weighted norm estimate for $\boldsymbol{\theta}^{\prime}$ which, together with a maximum norm estimate for the stationary problem, implies an essentially optimal order uniform error estimate for (3.1) away from $x=0$.

THEOREM 3. Let $u$ be the solution of (1.1) and $u_{h}$ that of (3.1) with $v_{h}=P_{A} v$. Then for any $\alpha \in(0,1)$ there is a constant $C_{\alpha}$ such that

$$
\left\|u_{h}(t)-u(t)\right\|_{L_{x}(\alpha, 1)} \leqslant C_{\alpha} h^{r}\left\{\left(\ln \frac{1}{h}\right)^{\bar{r}}\left\|u^{(r)}(t)\right\|_{L_{x}}+\left(\int_{0}^{t}\left\|x^{b / 2} u_{t}^{(r)}\right\|^{2} d s\right)^{1 / 2}\right\},
$$

where $\bar{r}=1$ if $r=2$ and $\bar{r}=0$ if $r>2$.

Proof. With the notation of Theorem 2 we have by the maximum norm estimate (1.5) for the stationary problem

$$
\|\rho(t)\|_{L_{\infty}} \leqslant C h^{r}\left(\ln \frac{1}{h}\right)^{\bar{r}}\left\|u^{(r)}(t)\right\|_{L_{\infty}} .
$$

Further, by obvious estimates

$$
\|\theta\|_{L_{\infty}(\alpha, 1)} \leqslant\left\|\theta^{\prime}\right\|_{L_{1}(\alpha, 1)} \leqslant C(\alpha, b)\left\|x^{b / 2} \theta^{\prime}\right\|,
$$

and hence the appropriate estimate for the latter quantity will complete the proof. Setting $\chi=\theta_{t}$ in (3.4), we have

$$
\left\|x^{b / 2} \theta_{t}\right\|^{2}+\frac{1}{2} \frac{d}{d t} A(\theta, \theta)=-\left(x^{b} \rho_{t}, \theta_{t}\right)<\frac{1}{2}\left\|x^{b / 2} \rho_{t}\right\|^{2}+\frac{1}{2}\left\|x^{b / 2} \theta_{t}\right\|^{2},
$$

so that in view of Theorem 1

$$
\frac{d}{d t} A(\theta, \theta) \leqslant\left\|x^{b / 2} \rho_{t}\right\|^{2} \leqslant C h^{2 r}\left\|x^{b / 2} u_{t}^{(r)}\right\|^{2}
$$

and hence by integration, since $\theta(0)=0$,

$$
\left\|x^{b / 2} \theta^{\prime}(t)\right\|^{2} \leqslant A(\theta(t), \theta(t)) \leqslant C h^{2 r} \int_{0}^{t}\left\|x^{b / 2} u_{t}^{(r)}\right\|^{2} d s .
$$

This completes the proof.

Remark. For $0 \leqslant b<1$ we may take $\alpha=0$ in Theorem 3 and thus obtain (essentially) optimal order global uniform convergence of the approximate solution.

4. The Nonsymmetric Method. For $b>1$ we shall now analyze the approximate solution in $S_{h}$ of the stationary problem (1.1) defined by

$$
B\left(u_{h}, \chi\right)=(x f, \chi) \quad \forall \chi \in S_{h},
$$

where $B(\cdot, \cdot)$ is the nonsymmetric bilinear form

$$
B(v, w)=\left(x v^{\prime}, w^{\prime}\right)-(b-1)\left(v^{\prime}, w\right)+(x q v, w) .
$$

In view of the corresponding variational equations for the exact solution of (1.1) we have

$$
B\left(u_{h}-u, \chi\right)=0 \quad \forall \chi \in S_{h},
$$

and using this we shall devote the rest of this section to the proof of the following optimal order uniform error estimate. 
THEOREM 4. With $b>1$, let $u$ be the solution of (1.1) and $u_{h}$ that of (4.1). Then

$$
\left\|u_{h}-u\right\|_{L_{\infty}} \leqslant C h^{r}\left\|u^{(r)}\right\|_{L_{\infty}} .
$$

The essential steps in the proof of this theorem are the following two lemmas.

LEMMA 3. Under the assumptions of Theorem 4,

$$
\left\|u_{h}-u\right\|_{L_{\infty}} \leqslant C h\left\|u_{h}^{\prime}-u^{\prime}\right\|_{L_{\infty}} .
$$

Lemma 4. Let $P_{B}$ be the elliptic projection onto $S_{h}$ defined by

$$
B\left(P_{B} v-v, \chi\right)=0 \quad \forall \chi \in S_{h} .
$$

Then, for $v$ vanishing at $x=1$,

$$
\left\|\left(P_{B} v\right)^{\prime}\right\|_{L_{\infty}} \leqslant C\left\|v^{\prime}\right\|_{L_{\infty}} .
$$

Before we prove these lemmas we shall show how the theorem follows.

Proof of Theorem 4. Note that, for $\chi \in S_{h}$,

$$
u_{h}-u=P_{B}(u-\chi)-(u-\chi),
$$

so that, by Lemma 4 and the standard approximation property of $S_{h}$,

$$
\left\|u_{h}^{\prime}-u^{\prime}\right\|_{L_{\infty}} \leqslant C \inf _{\chi \in S_{h}}\left\|u^{\prime}-\chi^{\prime}\right\|_{L_{x}} \leqslant C h^{r-1}\left\|u^{(r)}\right\|_{L_{\infty}} .
$$

The result now follows by Lemma 3 .

We shall now prove Lemmas 3 and 4.

Proof of Lemma 3. Assuming that the maximal error is attained at the point $x \in \bar{I}_{i}$, we have

$$
\begin{aligned}
\left\|u_{h}-u\right\|_{L_{x}} & =\left|\left(u_{h}-u\right)\left(x_{i}\right)+\int_{x_{i}}^{x}\left(u_{h}^{\prime}-u^{\prime}\right) d s\right| \\
& \leqslant\left|\left(u_{h}-u\right)\left(x_{i}\right)\right|+h\left\|u_{h}^{\prime}-u^{\prime}\right\|_{L_{x}}
\end{aligned}
$$

and it hence suffices to estimate $u_{h}-u$ at the interior mesh points $x_{i}, i=1, \ldots$, $N-1$. For this we shall use the Green's function $g=g_{x_{i}}$ vanishing at $x=0$ and $x=1$ and satisfying

$$
B(v, g)=v\left(x_{i}\right) \quad \forall v \in W_{\infty}^{1} \text { with } v(1)=0,
$$

or, with $\delta$ the Dirac delta function,

$$
-x g^{\prime \prime}+(b-2) g^{\prime}+x q(x) g=\delta\left(x-x_{i}\right), \quad g(0)=g(1)=0 .
$$

We shall show that this problem has a unique solution $g$ such that

$$
\left\|x g^{\prime \prime}\right\|_{L_{1}^{h}} \equiv \sum_{j=1}^{N}\left\|x g^{\prime \prime}\right\|_{L_{1}\left(I_{j}\right)} \leqslant C,
$$

where the constant is bounded independently of $i$ for fixed $b$ and $q$.

Assuming this for a moment and using (4.2) and Lemma 1, we have

$$
\begin{aligned}
\left|\left(u_{h}-u\right)\left(x_{i}\right)\right| & =\left|B\left(u_{h}-u, g-\chi\right)\right| \\
& \leqslant C\left\|u_{h}^{\prime}-u^{\prime}\right\|_{L_{\infty}}\left(\left\|x\left(g^{\prime}-\chi^{\prime}\right)\right\|_{L_{1}}+\|g-\chi\|_{L_{1}}\right) \quad \forall \chi \in S_{h} .
\end{aligned}
$$


We shall prove the following

Proposition 1. There is a constant $C=C(\beta)$ and for each appropriately regular $v$ vanishing at $x=1$ an interpolant $\bar{v} \in S_{h}$ such that

$$
\left\|x\left(v^{\prime}-\bar{v}^{\prime}\right)\right\|_{L_{1}}+\|v-\vec{v}\|_{L_{1}} \leqslant C h\left\|x v^{\prime \prime}\right\|_{L_{1}^{h}} .
$$

Clearly, the proposition together with (4.7) completes the proof of Lemma 3.

To prove the proposition, let $\bar{v}$ be the piecewise linear function which interpolates $v$ at the points $x_{j}, j=1, \ldots, N$, and which is determined on $I_{1}$ by the additional requirement that $\bar{v}^{\prime}\left(x_{1}\right)=v^{\prime}\left(x_{1}\right)$. We then have the standard estimate

$$
\|v-\vec{v}\|_{L_{1}\left(I_{j}\right)}+h_{j}\left\|v^{\prime}-\bar{v}^{\prime}\right\|_{L_{1}\left(I_{j}\right)} \leqslant C h_{j}^{2}\left\|v^{\prime \prime}\right\|_{L_{1}\left(I_{j}\right)}, \quad j=1, \ldots, N,
$$

and hence, for all intervals except $I_{1}$,

$$
\left\|x\left(v^{\prime}-\bar{v}^{\prime}\right)\right\|_{L_{1}\left(I_{j}\right)}+\|v-\bar{v}\|_{L_{1}\left(I_{j}\right)} \leqslant C h\left\|x v^{\prime \prime}\right\|_{L_{1}\left(I_{j}\right)} .
$$

In order to show the analogous estimate on $I_{1}$ we note that, for $x \in I_{1}$,

$$
\left|x\left(v^{\prime}-\bar{v}^{\prime}\right)(x)\right|=\left|x \int_{x}^{x_{1}} s^{-1} s v^{\prime \prime}(s) d s\right| \leqslant\left\|x v^{\prime \prime}\right\|_{L_{1}\left(I_{1}\right)},
$$

so that

$$
\left\|x\left(v^{\prime}-\bar{v}^{\prime}\right)\right\|_{L_{1}\left(I_{1}\right)} \leqslant h\left\|x v^{\prime \prime}\right\|_{L_{1}\left(I_{1}\right)} .
$$

Further, since both $v-\bar{v}$ and its first derivative vanish at $x_{1}$, we have

$$
|(v-\bar{v})(x)|=\left|\int_{x}^{x_{1}}(s-x) v^{\prime \prime}(s) d s\right| \leqslant\left\|x v^{\prime \prime}\right\|_{L_{1}\left(I_{1}\right)},
$$

and hence

$$
\|v-\vec{v}\|_{L_{1}\left(I_{1}\right)} \leqslant h\left\|x v^{\prime \prime}\right\|_{L_{1}\left(I_{1}\right)},
$$

which completes the proof of $(4.8)$ for $j=1$. The proposition now follows at once by summation of (4.8).

It remains to show that (4.5) admits a solution with the properties stated. Recall that $b>1$ and define

$$
\gamma(x, y)= \begin{cases}\frac{1}{b-1}\left(y^{-(b-1)}-1\right) x^{b-1} & \text { for } y \geqslant x \\ \frac{1}{b-1}\left(1-x^{b-1}\right) & \text { for } y<x\end{cases}
$$

In the special case $q=0$ the solution of (4.5) is $g_{x_{i}}=\gamma\left(\cdot, x_{i}\right)$, and the verification of its regularity properties is straightforward. In the general case we may formulate (4.5) (or (4.6)) as the integral equation

$$
g+K g=\gamma\left(x, x_{i}\right)
$$

where $K$ is the integral operator defined by

$$
K \varphi(x)=\int_{0}^{1} \gamma(x, y) y q(y) \varphi(y) d y .
$$

Since $\gamma$ is uniformly bounded and $\left\|\gamma_{x}^{\prime}(x, \cdot)\right\|_{L_{1}}$ is bounded, uniformly in $x$, it follows by Arzelà-Ascoli's theorem that $K$ is compact on $C(\bar{I})$. Hence (4.9) admits a unique 
solution in $C(\bar{I})$ as a result of the fact that the corresponding homogeneous equation

$$
B(v, \varphi)=0 \quad \forall v \in W_{\infty}^{1} \text { with } v(1)=0,
$$

has only the trivial solution, since $B(\cdot, \cdot)$ is positive. It follows that $(I+K)^{-1}$ is bounded on $C(\bar{I})$ so that, in particular,

$$
\|g\|_{L_{\infty}} \leqslant C\|\gamma\|_{L_{\infty}}=C \frac{1-x_{i}^{b-1}}{b-1} \leqslant C .
$$

In order to complete the proof we note from (4.6) that

$$
\left\|x g^{\prime \prime}\right\|_{L_{1}^{h}} \leqslant C\left(\left\|g^{\prime}\right\|_{L_{1}}+\|g\|_{L_{1}}\right) \leqslant C\left\|g^{\prime}\right\|_{L_{1}},
$$

and that from (4.9) and (4.10)

$$
\left\|g^{\prime}\right\|_{L_{1}} \leqslant\left\|\gamma^{\prime}\right\|_{L_{1}}+\left\|(K g)^{\prime}\right\|_{L_{1}} \leqslant C\left\|\gamma^{\prime}\right\|_{L_{1}}\left(1+\|g\|_{L_{\infty}}\right) \leqslant C .
$$

This completes the proof of (4.7) and hence of Lemma 3.

We now turn to the

Proof of Lemma 4. We shall prove that for $v_{h}=P_{B} v$ we have

$$
\left\|v_{h}^{\prime}\right\|_{L_{\infty}} \leqslant C\left\|v^{\prime}\right\|_{L_{\infty}} \text {. }
$$

We introduce the principal part of $B(\cdot, \cdot)$,

$$
B_{0}(v, w)=\left(v^{\prime}, x w^{\prime}-(b-1) w\right),
$$

and write (4.3) in the form

$$
B_{0}\left(v_{h}, \chi\right)=B_{0}(v, \chi)-\left(x q\left(v_{h}-v\right), \chi\right) \quad \forall \chi \in S_{h} .
$$

We now introduce a basis $\left\{\varphi_{i k} ; i=1, \ldots, N, k=1, \ldots, r-1\right\}$ for the trial functions by

$$
\varphi_{i k}(x)= \begin{cases}-h_{i} & \text { for } x \leqslant x_{i-1}, \\ (-1)^{k-1} h_{i}\left(\left(x-x_{i}\right) / h_{i}\right)^{k} & \text { for } x_{i-1}<x \leqslant x_{i}, \\ 0 & \text { for } x_{i}<x\end{cases}
$$

and set

$$
v_{h}=\sum_{i, k} v_{i k} \varphi_{i k} .
$$

Since the derivatives of the $\varphi_{i k}$ are uniformly bounded, we have

$$
\left\|v_{h}^{\prime}\right\|_{L_{\infty}} \leqslant C \max _{i, k}\left|v_{i k}\right|
$$

and it thus suffices to bound each of the $v_{i k}$ appropriately. By (4.12) these components satisfy

$$
\sum_{i, k} v_{i k} B_{0}\left(\varphi_{i k}, \chi\right)=B_{0}(v, \chi)-\left(x q\left(v_{h}-v\right), \chi\right) \quad \forall \chi \in S_{h} .
$$

We shall now construct a basis $\left\{\psi_{j l} ; j=1, \ldots, N, l=1, \ldots, r-1\right\}$ for the test functions such that

$$
B_{0}\left(\varphi_{i k}, \psi_{j l}\right)=0 \text { for } i \neq j, 1 \leqslant k, l \leqslant r-1,
$$

such that the matrix $B_{j}=\left(B_{0}\left(\varphi_{j k}, \psi_{j l}\right)\right)$ is nonsingular, with

$$
\left\|B_{j}^{-1}\right\|_{l_{\infty}} \leqslant C\left(x_{j} h_{j}\right)^{-1}
$$


and finally such that

$$
\left\|x \psi_{j l}^{\prime}\right\|_{L_{1}}+\left\|\psi_{j l}\right\|_{L_{1}} \leqslant C x_{j} h_{j} .
$$

Assuming such a basis $\left\{\psi_{j l}\right\}$ already at our disposal, we observe from (4.14) that the system (4.13) now splits into one smaller system for each interval $I_{j}$, namely, with $(\cdot)$ denoting column vectors,

$$
B_{j}\left(v_{j k}\right)=\left(B_{0}\left(v, \psi_{j l}\right)-\left(x q\left(v_{h}-v\right), \psi_{j l}\right)\right) .
$$

Solving for $v_{j k}$ we obtain, using (4.15) and (4.16),

$$
\begin{aligned}
\left|v_{j k}\right| & \leqslant\left\|B_{j}^{-1}\right\|_{L_{\infty}} \max _{l}\left|B_{0}\left(v, \psi_{j l}\right)-\left(x q\left(v_{h}-v\right), \psi_{j l}\right)\right| \\
& \leqslant C x_{j}^{-1} h_{j}^{-1} \max _{l}\left\{\left\|v^{\prime}\right\|_{L_{\infty}}\left\|x \psi_{j l}^{\prime}-(b-1) \psi_{j l}\right\|_{L_{1}}+\left\|v_{h}-v\right\|_{L_{\infty}}\left\|\psi_{j l}\right\|_{L_{1}}\right\} \\
& \leqslant C\left\{\left\|v^{\prime}\right\|_{L_{\infty}}+\left\|v_{h}-v\right\|_{L_{\infty}}\right\} .
\end{aligned}
$$

We now apply Lemma 3 to estimate the last term and obtain

$$
\left\|v_{h}^{\prime}\right\|_{L_{\infty}} \leqslant C\left\|v^{\prime}\right\|_{L_{\infty}}+C h\left\|v_{h}^{\prime}-v^{\prime}\right\|_{L_{\infty}} \leqslant C\left\|v^{\prime}\right\|_{L_{\infty}}+C h\left\|v_{h}^{\prime}\right\|_{L_{\infty}} \text {. }
$$

Since $h$ may be considered small, this implies the desired estimate (4.11).

We now turn to the construction of the $\psi_{j l}$. This will be accomplished by a modification of the $\varphi_{j l}$ in $\left(0, x_{j-1}\right)$, or by setting

$$
\psi_{j l}(x)=\varphi_{j l}(x)+\sum_{\substack{p<j \\ q}} b_{j l, p q} \varphi_{p q}(x),
$$

with $b_{j l, p q}$ suitably chosen. We thus think of $j$ and $l$ as fixed and note at once that (4.14) automatically holds for $i>j$ since then $\varphi_{i k}^{\prime}$ vanishes on the support of $\psi_{j l}$. We also note that $B_{0}\left(\varphi_{j k}, \psi_{j l}\right)=B_{0}\left(\varphi_{j k}, \varphi_{j l}\right)$ and hence, with $\langle\cdot, \cdot\rangle$ the usual inner product in $R^{r-1}$, and $y=\sum_{k} \eta_{k} \varphi_{j k}, \eta=\left(\eta_{1}, \ldots, \eta_{r-1}\right) \in R^{r-1}$,

$$
\left\langle B_{j} \eta, \eta\right\rangle=B_{0}(y, y) \geqslant \int_{I_{j}} x y^{\prime 2} d x \geqslant C x_{j} h_{j} \sum_{k} \eta_{k}^{2},
$$

where we have used a scaling argument and the equivalence of different norms on $P_{r-1}(I)$ in the last step. In view of the equivalence of different norms on $R^{r-1}$, we conclude in particular that (4.15) holds. Now consider the case $i<j$ in (4.14). By our definition of $\psi_{j l}$ and with the coefficients $b_{p q} \equiv b_{j l, p q}$ at our disposal we shall obtain

$$
B_{0}\left(\varphi_{i k},-h_{j}+\sum_{\substack{p<j \\ q}} b_{p q} \varphi_{p q}\right)=0 \quad \text { for } i<j, 1 \leqslant k \leqslant r-1 .
$$

Rearranging terms and using that $B_{0}\left(\varphi_{i k}, \varphi_{p q}\right)$ vanishes for $p<i$, we write this as

$$
\sum_{q} b_{i q} B_{0}\left(\varphi_{i k}, \varphi_{i q}\right)=B_{0}\left(\varphi_{i k}, h_{j}-\sum_{\substack{i<p<j \\ q}} b_{p q} \varphi_{p q}\right) \text { for } i<j, 1 \leqslant k \leqslant r-1 \text {. }
$$

Since the matrices $B_{i}=\left(B_{0}\left(\varphi_{i k}, \varphi_{i q}\right)\right)$ are invertible, we can solve this system successively for $i=j-1, j-2, \ldots, 1$. We have thus proved that the construction of a basis $\left\{\psi_{j l}\right\}$ satisfying (4.14) is indeed possible, and we shall proceed now to prove also (4.16). 
Since $\psi_{j l}$ equals $\varphi_{j l}$ on $\left(x_{j-1}, 1\right)$, we have at once

$$
\left\|x \psi_{j l}^{\prime}\right\|_{L_{1}\left(x_{j-1}, 1\right)}+\left\|\psi_{j l}\right\|_{L_{1}\left(x_{j-1}, 1\right)} \leqslant C x_{j} h_{j},
$$

so it remains to show the appropriate estimate on $\left(0, x_{j-1}\right)$.

For transparency we shall write $\psi$ for $\psi_{j l}, \varphi$ for $\varphi_{j l}$, and $J$ for $\left(0, x_{j-1}\right)$ in the ensuing computation. We have

$$
\left\|x \psi^{\prime}\right\|_{L_{1}(J)} \leqslant\left\|x^{1 / 2}\right\|_{L_{2}(J)}\left\|x^{1 / 2} \psi^{\prime}\right\|_{L_{2}(J)} \leqslant C x_{j}\left\|x^{1 / 2} \psi^{\prime}\right\|_{L_{2}(J)},
$$

and further

$$
\begin{aligned}
|\psi(x)| & =\left|-h_{j}-\int_{x}^{x_{j-1}} s^{-1 / 2} s^{1 / 2} \psi^{\prime}(s) d s\right| \\
& \leqslant h_{j}+\left\|x^{-1 / 2}\right\|_{L_{2}\left(x, x_{j-1}\right)}\left\|x^{1 / 2} \psi^{\prime}\right\|_{L_{2}(J)} \leqslant h_{j}+\left(\ln \left(x_{j-1} / x\right)\right)^{1 / 2}\left\|x^{1 / 2} \psi^{\prime}\right\|_{L_{2}(J)},
\end{aligned}
$$

and hence

$$
\left\|x \psi^{\prime}\right\|_{L_{1}(J)}+\|\psi\|_{L_{1}(J)} \leqslant C x_{j} h_{j}+C x_{j}\left\|x^{1 / 2} \psi^{\prime}\right\|_{L_{2}(J)} .
$$

It remains to show that

$$
\left\|x^{1 / 2} \psi^{\prime}\right\|_{L_{2}(J)} \leqslant C h_{j}
$$

Using the equation of $\psi$ and (4.14), we have

$$
B_{0}(\psi, \psi)=B_{0}(\varphi, \psi)+\sum_{\substack{p<j \\ q}} b_{p q} B_{0}\left(\varphi_{p q}, \psi\right)=B_{0}(\varphi, \varphi)
$$

Since

$$
\begin{aligned}
B_{0}(\psi, \psi) & =\left(x \psi^{\prime}, \psi^{\prime}\right)-(b-1)\left(\psi^{\prime}, \psi\right) \\
& =\left\|x^{1 / 2} \psi^{\prime}\right\|_{L_{2}(J)}^{2}+\int_{I_{j}} x \varphi^{\prime} \varphi^{\prime} d x+\frac{b-1}{2} \psi^{2}(0)
\end{aligned}
$$

and since

$$
B_{0}(\varphi, \varphi)=\int_{I_{j}} x \varphi^{\prime} \varphi^{\prime} d x+\frac{b-1}{2} \varphi^{2}\left(x_{j-1}\right)
$$

we obtain

$$
\left\|x^{1 / 2} \psi^{\prime}\right\|_{L_{2}(J)}^{2}=\frac{b-1}{2} \varphi^{2}\left(x_{j-1}\right)-\frac{b-1}{2} \psi^{2}(0) \leqslant \frac{b-1}{2} \varphi^{2}\left(x_{j-1}\right) \leqslant C h_{j}^{2} .
$$

This shows (4.17) and thus completes the proof of Lemma 4.

5. The Nonsymmetric Method for the Time-Dependent Problem. For the case $b>1$ we shall now also consider the semidiscrete problem

$$
\begin{gathered}
\left(x u_{h, t}, \chi\right)+B\left(u_{h}, \chi\right)=(x f, \chi) \quad \forall \chi \in S_{h}, t>0, \\
u_{h}(0)=v_{h},
\end{gathered}
$$

where $B(\cdot, \cdot)$ is the nonsymmetric bilinear form defined in (1.7). Recalling the definition of the corresponding elliptic projection $P_{B}$ onto $S_{h}$,

$$
B\left(P_{B} v-v, \chi\right)=0 \quad \forall \chi \in S_{h},
$$

we shall first demonstrate the following uniform error estimate. 
THEOREM 5. Assume that $b>1$, and let $u$ be the solution of (1.2) and $u_{h}$ that of (5.1) with $v_{h}=P_{B} v$. Then

$$
\left\|u_{h}(t)-u(t)\right\|_{L_{\infty}} \leqslant C\left(\ln \frac{1}{h}\right)^{1 / 2} h^{r}\left\{\left\|u^{(r)}(t)\right\|_{L_{\infty}}+\left\|u_{t}^{(r)}(0)\right\|_{L_{\infty}}+\int_{0}^{t}\left\|u_{t t}^{(r)}\right\|_{L_{\infty}} d s\right\} .
$$

Proof. We write

$$
u_{h}-u=\left(u_{h}-P_{B} u\right)+\left(P_{B} u-u\right)=\theta+\rho,
$$

and recall first that, by Theorem 4 ,

$$
\|\rho(t)\|_{L_{\infty}} \leqslant C h^{r}\left\|u^{(r)}(t)\right\|_{L_{\infty}}
$$

so that it only remains to estimate $\theta$. For this we shall show below that, for any $\chi \in S_{h}$,

$$
\|\chi\|_{L_{\infty}} \leqslant\left\|\chi^{\prime}\right\|_{L_{1}} \leqslant C\left(\ln \frac{1}{h}\right)^{1 / 2}\left\|x^{1 / 2} \chi^{\prime}\right\|
$$

and to complete the proof, we shall apply (5.3) to $\chi=\theta$ and show that

$$
\left\|x^{1 / 2} \theta^{\prime}(t)\right\| \leqslant C h^{r}\left\{\left\|u_{t}^{(r)}(0)\right\|_{L_{\infty}}+\int_{0}^{t}\left\|u_{t t}^{(r)}\right\|_{L_{\infty}} d s\right\} .
$$

The first inequality of (5.3) is obvious, since $\chi(1)=0$. For the second we have, using first the equivalence of the $L_{1}$ norms over $\left(0, x_{1}\right)$ and $\left(x_{1} / 2, x_{1}\right)$ for functions in $S_{h}$, and then Schwarz' inequality,

$$
\begin{aligned}
\left\|\chi^{\prime}\right\|_{L_{1}} & \leqslant C\left\|\chi^{\prime}\right\|_{L_{1}\left(x_{1} / 2,1\right)} \leqslant C\left\|x^{-1 / 2}\right\|_{L_{2}\left(x_{1} / 2,1\right)}\left\|x^{1 / 2} \chi^{\prime}\right\|_{L_{2}\left(x_{1} / 2,1\right)} \\
& \leqslant C\left(\ln \frac{1}{h}\right)^{1 / 2}\left\|x^{1 / 2} \chi^{\prime}\right\| .
\end{aligned}
$$

In order to prove (5.4) we note that by (5.1), its analogue for (1.2), and (5.2)

$$
\left(x \theta_{t}, \chi\right)+B(\theta, \chi)=-\left(x \rho_{t}, \chi\right) \quad \forall \chi \in S_{h}, t>0 .
$$

With $\chi=\theta$ this yields

$$
\left\|x^{1 / 2} \theta^{\prime}\right\|^{2} \leqslant B(\theta, \theta) \leqslant\left(\left\|x^{1 / 2} \theta_{t}\right\|+\left\|x^{1 / 2} \rho_{t}\right\|\right)\left\|x^{1 / 2} \theta\right\|,
$$

and hence, by Lemma 1 ,

$$
\left\|x^{1 / 2} \theta^{\prime}\right\| \leqslant\left\|x^{1 / 2} \theta_{t}\right\|+\left\|x^{1 / 2} \rho_{t}\right\| .
$$

Here, from Theorem 4,

$$
\left\|x^{1 / 2} \rho_{t}(t)\right\| \leqslant\left\|\rho_{t}(t)\right\|_{L_{\infty}} \leqslant C h^{r}\left\{\left\|u_{t}^{(r)}(0)\right\|_{L_{\infty}}+\int_{0}^{t}\left\|u_{t t}^{(r)}\right\|_{L_{x}} d s\right\} .
$$

In order to estimate the term in $\theta_{t}$, we differentiate (5.5) and set $\chi=\theta_{t}$ to obtain

$$
\left(x \theta_{t t}, \theta_{t}\right)+B\left(\theta_{t}, \theta_{t}\right)=-\left(x \rho_{t t}, \theta_{t}\right) \text { for } t>0,
$$

whence

$$
\left\|x^{1 / 2} \theta_{t}\right\| \frac{d}{d t}\left\|x^{1 / 2} \theta_{t}\right\| \leqslant\left\|x^{1 / 2} \rho_{t t}\right\|\left\|x^{1 / 2} \theta_{t}\right\|
$$

and

$$
\left\|x^{1 / 2} \theta_{t}(t)\right\| \leqslant\left\|x^{1 / 2} \theta_{t}(0)\right\|+\int_{0}^{t}\left\|x^{1 / 2} \rho_{t t}\right\| d s .
$$


Since $\theta(0)=0$, we obtain from (5.5) with $\chi=\theta_{t}(0)$

$$
\left\|x^{1 / 2} \theta_{t}(0)\right\|^{2}=-\left(x \rho_{t}(0), \theta_{t}(0)\right) \leqslant\left\|x^{1 / 2} \rho_{t}(0)\right\|\left\|x^{1 / 2} \theta_{t}(0)\right\|,
$$

so that

$$
\left\|x^{1 / 2} \theta_{t}(0)\right\| \leqslant\left\|\rho_{t}(0)\right\|_{L_{\infty}} \leqslant C h^{r}\left\|u_{t}^{(r)}(0)\right\|_{L_{\infty}} .
$$

Finally, again by Theorem 4 ,

$$
\int_{0}^{t}\left\|x^{1 / 2} \rho_{t t}\right\| d s \leqslant \int_{0}^{t}\left\|\rho_{t t}\right\|_{L_{\infty}} d s \leqslant C h^{r} \int_{0}^{t}\left\|u_{t t}^{(r)}\right\|_{L_{\infty}} d s
$$

Together, (5.6), (5.7), (5.8), (5.9), and (5.10) show (5.4) and thus complete the proof.

In the proof of the above result the discrete initial data had to be chosen as $v_{h}=P_{B} v$. We shall now show, using a smoothing property of the discrete solution operator, that any optimal order initial approximation will produce a discrete solution which is of essentially optimal order in the uniform norm for $t$ positive.

THEOREM 6. Let $u$ and $u_{h}$ be the solutions of (1.2) and (5.1), respectively. Then

$$
\begin{aligned}
\left\|u_{h}(t)-u(t)\right\|_{L_{\infty}} \leqslant & C t^{-1 / 2} \ln \frac{1}{h}\left\|x^{1 / 2}\left(P_{B} v-v_{h}\right)\right\| \\
& +C\left(\ln \frac{1}{h}\right)^{1 / 2} h^{r}\left\{\left\|u^{(r)}(t)\right\|_{L_{\infty}}+\left\|u_{t}^{(r)}(0)\right\|_{L_{\infty}}+\int_{0}^{t}\left\|u_{t t}^{(r)}\right\|_{L_{\infty}} d s\right\} .
\end{aligned}
$$

Proof. Let $\tilde{u}_{h}(t)$ be the solution of the semidiscrete problem (5.1) with $v_{h}=P_{B} v$, and let $\eta=\tilde{u}_{h}-u_{h}$. Then, in view of (5.3) and Theorem 5, it suffices to show that

$$
\left\|x^{1 / 2} \eta^{\prime}(t)\right\| \leqslant C t^{-1 / 2}\left(\ln \frac{1}{h}\right)^{1 / 2}\left\|x^{1 / 2} \eta(0)\right\|,
$$

where $\eta$ satisfies the homogeneous equations

$$
\left(x \eta_{t}, \chi\right)+B(\eta, \chi)=0 \quad \forall \chi \in S_{h}, t>0 .
$$

With $\chi=\eta$ we have

$$
\left\|x^{1 / 2} \eta^{\prime}\right\|^{2} \leqslant B(\eta, \eta)=-\left(x \eta_{t}, \eta\right) \leqslant\left\|x^{1 / 2} \eta_{t}\right\|\left\|x^{1 / 2} \eta\right\| .
$$

It follows at once from (5.11) that

$$
\frac{1}{2} \frac{d}{d t}\left\|x^{1 / 2} \eta\right\|^{2}+B(\eta, \eta)=0
$$

and hence

$$
\left\|x^{1 / 2} \eta(t)\right\|^{2}+2 \int_{0}^{t} B(\eta, \eta) d s=\left\|x^{1 / 2} \eta(0)\right\|^{2},
$$

which bounds, in particular, the second factor in (5.12). The proof will be completed by showing that

$$
\left\|x^{1 / 2} \eta_{t}(t)\right\| \leqslant C t^{-1} \ln \frac{1}{h}\left\|x^{1 / 2} \eta(0)\right\| .
$$

In the proof of this we shall need the boundedness of $B(\cdot, \cdot)$ on $S_{h}$, or

$$
\begin{aligned}
B(\chi, \zeta) & =\left(x \chi^{\prime}, \zeta^{\prime}\right)-(b-1)\left(\chi^{\prime}, \zeta\right)+(x q \chi, \zeta) \\
& \leqslant C \ln \frac{1}{h} B(\chi, \chi)^{1 / 2} B(\zeta, \zeta)^{1 / 2} \quad \forall \chi, \zeta \in S_{h},
\end{aligned}
$$


which follows easily if we note that, by (5.3),

$$
\left|\left(\chi^{\prime}, \zeta\right)\right| \leqslant\left\|\chi^{\prime}\right\|_{L_{1}}\|\zeta\|_{L_{\infty}} \leqslant C \ln \frac{1}{h}\left\|x^{1 / 2} \chi^{\prime}\right\|\left\|x^{1 / 2} \zeta^{\prime}\right\| .
$$

We now proceed with the proof of (5.14). We have from (5.11)

$$
\frac{d}{d t}\left(t^{2}\left\|x^{1 / 2} \eta_{t}\right\|^{2}\right)+2 t^{2} B\left(\eta_{t}, \eta_{t}\right)=2 t\left\|x^{1 / 2} \eta_{t}\right\|^{2}
$$

Here, using (5.15),

$$
\left\|x^{1 / 2} \eta_{t}\right\|^{2}=-B\left(\eta, \eta_{t}\right) \leqslant C \ln \frac{1}{h} B(\eta, \eta)^{1 / 2} B\left(\eta_{t}, \eta_{t}\right)^{1 / 2} .
$$

Hence

$$
2 t\left\|x^{1 / 2} \eta_{t}\right\|^{2} \leqslant 2 t^{2} B\left(\eta_{t}, \eta_{t}\right)+C\left(\ln \frac{1}{h}\right)^{2} B(\eta, \eta),
$$

so that, by integration of (5.16) and using (5.13),

$$
t^{2}\left\|x^{1 / 2} \eta_{t}\right\|^{2} \leqslant C\left(\ln \frac{1}{h}\right)^{2} \int_{0}^{t} B(\eta, \eta) d s \leqslant C\left(\ln \frac{1}{h}\right)^{2}\left\|x^{1 / 2} \eta(0)\right\|^{2} .
$$

This completes the proof.

6. Numerical Integration. In this section we shall discuss the computational solution of the nonsymmetric variational problem for the stationary problem, i.e., the equations

$$
B\left(u_{h}, \chi\right)=(x f, \chi) \quad \forall \chi \in S_{h} .
$$

With respect to some basis $\left\{\chi_{j}\right\}_{1}^{M}$ for $S_{h}$ we may write this system in the form

$$
\sum_{i=1}^{M} U_{i}\left(B_{0}\left(\chi_{i}, \chi_{j}\right)+\left(x q \chi_{i}, \chi_{j}\right)\right)=\left(x f, \chi_{j}\right), \quad j=1, \ldots, M .
$$

Except for simple choices of $q$ and $f$, the terms involving these functions will have to be approximated by numerical quadrature, and we shall see now how this can be done so as to maintain the convergence rate of the exact solution of (6.1).

Consider a quadrature formula

$$
Q(v) \equiv \sum_{s=1}^{n} \omega_{s} v\left(y_{s}\right) \sim \int_{0}^{1} v(x) d x \equiv I(v),
$$

with $\omega_{s} \geqslant 0$ and $y_{s} \in[0,1]$ and such that

$$
E(p) \equiv Q(p)-I(p)=0 \quad \forall p \in P_{k} .
$$

For example, the trapezoidal rule and Simpson's rule are of this form with $k=1$ and $k=3$, respectively, and so is the Gauss rule with $k=2 n-1$, if $n$ is the number of nodes. For the computation of the terms in $q$ and $f$ we shall employ a composite scheme using such a quadrature method on each of the intervals $I_{i}$, or

$$
\tilde{Q}(v)=\sum_{i=1}^{N} h_{i} Q\left(v\left(x_{i-1}+h_{i} \cdot\right)\right)=\sum_{i=1}^{N} h_{i} \sum_{s=1}^{n} \omega_{s} v\left(x_{i-1}+h_{i} y_{s}\right),
$$

and define thus the approximate solution $\tilde{u}_{h} \in S_{h}$ by

$$
\tilde{B}\left(\tilde{u}_{h}, \chi\right) \equiv B_{0}\left(\tilde{u}_{h}, \chi\right)+\tilde{Q}\left(x q \tilde{u}_{h} \chi\right)=\tilde{Q}(x f \chi) \quad \forall \chi \in S_{h} .
$$


Since $q$ and the quadrature weights $\omega_{s}$ are all nonnegative, it follows that $\tilde{B}(\cdot, \cdot)$ is positive, so that, in particular, (6.2) admits a unique solution. We shall prove the following estimate for the error introduced by the quadrature:

THEOREM 7. With $k \geqslant r-2, b>1$ and $q \in W_{\infty}^{k+3-r}$, let $u_{h}$ and $\tilde{u}_{h}$ be the solutions of (6.1) and (6.2), respectively. Then

$$
\left\|\tilde{u}_{h}-u_{h}\right\|_{L_{\infty}} \leqslant C h^{k+3-r}\left\{\|f\|_{W_{\infty}^{k+3-r}}+\|u\|_{W_{\infty}^{k+3-r}}\right\} .
$$

Proof. Set $\theta=\tilde{u}_{h}-u_{h}$ and $F=f-q u_{h}$. We shall prove below that

$$
\|\boldsymbol{\theta}\|_{L_{\infty}} \leqslant C h\left\|\boldsymbol{\theta}^{\prime}\right\|_{L_{\infty}}+C h^{j}\|F\|_{W_{\infty}^{j, h},}, \quad 0 \leqslant j \leqslant k+1,
$$

and that

$$
\left\|\theta^{\prime}\right\|_{L_{x}} \leqslant C\|\theta\|_{L_{x}}+C h^{j}\|F\|_{W_{x}^{j, h}, \quad 0 \leqslant j \leqslant k+2-r .}
$$

Since we may consider $h$ small, we deduce from these estimates that

$$
\begin{aligned}
\|\theta\|_{L_{\infty}} & \leqslant C h^{k+3-r}\|F\|_{W_{\infty}^{k+3-r, h}} \\
& \leqslant C h^{k+3-r}\left\{\|f\|_{W_{\infty}^{k+3-r}}+\max _{1 \leqslant s \leqslant \min (r-1, k+3-r)}\left\|u_{h}^{(s)}\right\|_{L_{\infty}^{h}}\right\},
\end{aligned}
$$

where we have also used Lemma 1 and the fact that $u_{h}$ is a piecewise polynomial of degree at most $r-1$. Given $s$ with $1 \leqslant s \leqslant r$ we have for a suitable interpolant $\tilde{u} \in S_{h}$ of $u$, using the first part of (4.4),

$$
\begin{aligned}
\left\|u_{h}^{\prime}-\tilde{u}^{\prime}\right\|_{L_{\infty}} & \leqslant\left\|u_{h}^{\prime}-u^{\prime}\right\|_{L_{\infty}}+\left\|u^{\prime}-\tilde{u}^{\prime}\right\|_{L_{\infty}} \\
& \leqslant C\left\|u^{\prime}-\tilde{u}^{\prime}\right\|_{L_{\infty}} \leqslant C h^{s-1}\left\|u^{(s)}\right\|_{L_{\infty}},
\end{aligned}
$$

and since the interpolant may be taken to be a piecewise polynomial of degree at most $s-1$ and since inverse estimates hold, we obtain

$$
\left\|u_{h}^{(s)}\right\|_{L_{\infty}^{h}}=\left\|\left(u_{h}-\tilde{u}\right)^{(s)}\right\|_{L_{\infty}^{h}} \leqslant C h^{-(s-1)}\left\|u_{h}^{\prime}-\tilde{u}^{\prime}\right\|_{L_{\infty}} \leqslant C\left\|u^{(s)}\right\|_{L_{\infty}} .
$$

Except for the verification of (6.3) and (6.4) this completes the proof of the theorem.

For the proof of (6.3) we have first

$$
\|\theta\|_{L_{\infty}} \leqslant \max _{1 \leqslant i \leqslant N-1}\left|\theta\left(x_{i}\right)\right|+h\left\|\theta^{\prime}\right\|_{L_{\infty}} .
$$

With $g=g_{x_{i}}$ defined as in the proof of Lemma 3 and with $\bar{g} \in S_{h}$ the piecewise linear interpolant of $g$ defined by $\bar{g}\left(x_{i}\right)=g\left(x_{i}\right)$ for $i=1, \ldots, N$ and $\bar{g}^{\prime}\left(x_{1}^{-}\right)=g^{\prime}\left(x_{1}^{-}\right)$, we have

$$
\theta\left(x_{i}\right)=B(\theta, g)=B(\theta, g-\bar{g})+B(\theta, \bar{g}),
$$

and consequently, by the approximation properties of the interpolant stated in Proposition 1 of Section 4,

$$
\begin{aligned}
\left|\theta\left(x_{i}\right)\right| & \leqslant C\left\|\theta^{\prime}\right\|_{L_{\infty}}\left\{\left\|x\left(g^{\prime}-\bar{g}^{\prime}\right)\right\|_{L_{1}}+\|g-\bar{g}\|_{L_{1}}\right\}+|B(\theta, \bar{g})| \\
& \leqslant C h\left\|\theta^{\prime}\right\|_{L_{\infty}}+|B(\theta, \bar{g})| .
\end{aligned}
$$

It remains to estimate the latter term by the right-hand side of (6.3). We begin by a bound for $\bar{g}^{\prime}$. We have first, using an inverse estimate,

$$
\left\|\bar{g}^{\prime}\right\|_{L_{1}\left(I_{1}\right)} \leqslant C h^{-1}\|\bar{g}\|_{L_{1}\left(I_{1}\right)} \leqslant C\|\bar{g}\|_{L_{\infty}\left(I_{1}\right)} \leqslant C\|g\|_{L_{\infty}} \leqslant C\left\|g^{\prime}\right\|_{L_{1}},
$$


and hence, using Proposition 1 once more,

$$
\begin{aligned}
\left\|\bar{g}^{\prime}\right\|_{L_{1}} & \leqslant\left\|\bar{g}^{\prime}\right\|_{L_{1}\left(I_{1}\right)}+\left\|\bar{g}^{\prime}-g^{\prime}\right\|_{L_{1}\left(x_{1}, 1\right)}+\left\|g^{\prime}\right\|_{L_{1}\left(x_{1}, 1\right)} \\
& \leqslant C\left(\left\|g^{\prime}\right\|_{L_{1}}+\left\|x g^{\prime \prime}\right\|_{L_{1}^{h}}\right) \leqslant C .
\end{aligned}
$$

Introducing the notation

$$
\tilde{E}(v) \equiv \sum_{i=1}^{N} h_{i} E\left(v\left(x_{i-1}+h_{i} \cdot\right)\right)=\tilde{Q}(v)-I(v),
$$

we have by (6.1) and (6.2)

$$
B(\theta, \bar{g})=\tilde{E}(x F \bar{g})-\tilde{E}(x q \theta \bar{g}) .
$$

By the assumptions on the quadrature rule $Q$ we have

$$
|E(v)|=\inf _{p \in p_{k}}|E(v-p)| \leqslant C \inf _{p \in p_{k}}\|v-p\|_{L_{\infty}} \leqslant C\left\|v^{(j)}\right\|_{L_{\infty}}, \quad 0 \leqslant j \leqslant k+1,
$$

and hence

$$
\begin{aligned}
|\tilde{E}(v)| & \leqslant C \sum_{i=1}^{N} h_{i}\left\|\left(v\left(x_{i-1}+h_{i} \cdot\right)\right)^{(j)}\right\|_{L_{\infty}} \\
& \leqslant C h^{j} \sum_{i=1}^{N} h_{i}\left\|v^{(j)}\right\|_{L_{\infty}\left(I_{i}\right)}, \quad 0 \leqslant j \leqslant k+1 .
\end{aligned}
$$

Using also the fact that, for piecewise polynomials of degree at most $r-1$,

$$
\sum_{i=1}^{N} h_{i}\|p\|_{L_{\infty}\left(I_{i}\right)} \leqslant C\|p\|_{L_{1}},
$$

we thus obtain

$$
\begin{aligned}
|\tilde{E}(x F \bar{g})| & \leqslant C h^{j} \sum_{i=1}^{N} h_{i}\left\|(x F \bar{g})^{(j)}\right\|_{L_{\infty}\left(I_{i}\right)} \\
& \leqslant C h^{j}\|F\|_{W_{\infty}^{j . h}} \sum_{i=1}^{N} h_{i}\left(\left\|\bar{g}^{\prime}\right\|_{L_{\infty}\left(I_{i}\right)}+\|\bar{g}\|_{L_{\infty}\left(I_{i}\right)}\right) \\
& \leqslant C h^{j}\|F\|_{W_{\infty}^{j . h}}\left(\left\|\bar{g}^{\prime}\right\|_{L_{1}}+\|\bar{g}\|_{L_{1}}\right) \leqslant C h^{j}\|F\|_{W_{\infty}^{j . h}},
\end{aligned}
$$

and similarly

$$
|\tilde{E}(x q \theta \bar{g})| \leqslant C h\|\theta\|_{W_{\infty}^{1}}\|\bar{g}\|_{W_{1}^{1}} \leqslant C h\left\|\theta^{\prime}\right\|_{L_{\infty}} .
$$

Together these estimates now prove (6.3).

Next we shall prove (6.4). For this we adopt the ideas and notation of the proof of Lemma 4 and set $\theta=\sum_{j, k} \theta_{j k} \varphi_{j k}$ so that, in particular,

$$
\left\|\theta^{\prime}\right\|_{L_{\infty}} \leqslant C \max _{j, k}\left|\theta_{j k}\right|
$$

and so that for our special choice of test functions $\psi_{j l}$ and in view of (6.1) and (6.2) we have

$$
\begin{aligned}
\sum_{k} \theta_{j k} B_{0}\left(\varphi_{j k}, \psi_{j l}\right)=B_{0}\left(\theta, \psi_{j l}\right)=\tilde{E}\left(x F \psi_{j l}\right)-\tilde{Q}\left(x q \theta \psi_{j l}\right) & \\
& j=1, \ldots, N, l=1, \ldots, r-1 .
\end{aligned}
$$


Recalling the estimates for the inverse of $B_{j}=\left(B_{0}\left(\varphi_{j k}, \psi_{j l}\right)\right)$,

$$
\left\|B_{j}^{-1}\right\|_{l_{\infty}} \leqslant C\left(x_{j} h_{j}\right)^{-1}
$$

and for the test functions $\psi_{j l}$,

$$
\left\|x \psi_{j l}^{\prime}\right\|_{L_{1}}+\left\|\psi_{j l}\right\|_{L_{1}} \leqslant C x_{j} h_{j}
$$

it only remains to find the appropriate estimates for $\tilde{E}\left(x F \psi_{j l}\right)$ and $\tilde{Q}\left(x q \theta \psi_{j l}\right)$ in terms of $\psi_{j l}$ and $x \psi_{j l}^{\prime}$.

First, since $Q(\cdot)$ is bounded in $L_{\infty}$, we have

$$
\left|\tilde{Q}\left(x q \theta \psi_{j l}\right)\right| \leqslant C\|\theta\|_{L_{\infty}} \sum_{i=1}^{N} h_{i}\left\|\psi_{j l}\right\|_{L_{\infty}\left(I_{i}\right)} \leqslant C\|\theta\|_{L_{\infty}}\left\|\psi_{j l}\right\|_{L_{1}} .
$$

In order to estimate $\tilde{E}\left(x F \psi_{j l}\right)$ we have first

$$
\left|\tilde{E}\left(x F \psi_{j l}\right)\right| \leqslant C\|F\|_{L_{\infty}} \sum_{i=1}^{N} h_{i}\left\|\psi_{j l}\right\|_{L_{\infty}\left(I_{i}\right)} \leqslant C\|F\|_{L_{\infty}}\left\|\psi_{j l}\right\|_{L_{1}},
$$

which completes the proof of (6.4) in the case $k=r-2$. For $k>r-2$, set $\psi=\psi_{j l}$, and let $\eta$ be a piecewise constant interpolant of $x \psi$. Then, as before,

$$
\begin{aligned}
|\tilde{E}(F \eta)| & \leqslant C h^{j}\|F\|_{W_{\infty}^{j, h}} \sum_{i=1}^{N} h_{i}\|\eta\|_{L_{\infty}\left(I_{i}\right)} \\
& \leqslant C h^{j}\|F\|_{W_{\infty}^{j, h}} \sum_{i=1}^{N} h_{i}\|x \psi\|_{L_{\infty}\left(I_{i}\right)} \\
& \leqslant C h^{j}\|F\|_{W_{\infty}^{j, h}}\|\psi\|_{L_{1}} .
\end{aligned}
$$

Moreover, since $Q$ is exact on $P_{k}$, we have for any $p_{r} \in P_{r}$ (with $P_{-1} \equiv\{0\}$ )

$$
\begin{aligned}
\left|E\left(v p_{r}\right)\right| & =\inf _{p \in P_{k-r}}\left|E\left((v-p) p_{r}\right)\right| \leqslant C \inf _{p \in P_{k-r}}\|v-p\|_{L_{\infty}}\left\|p_{r}\right\|_{L_{\infty}} \\
& \leqslant C\left\|v^{(j)}\right\|_{L_{\infty}}\left\|p_{r}\right\|_{L_{\infty}}, \quad 0 \leqslant j \leqslant k+1-r,
\end{aligned}
$$

and therefore

$$
|\tilde{E}(F(x \psi-\eta))| \leqslant C h^{j}\|F\|_{W_{\infty}^{j, h}} \sum_{i=1}^{N} h_{i}\|x \psi-\eta\|_{L_{\infty}\left(I_{i}\right)}, \quad 0 \leqslant j \leqslant k+1-r,
$$

so that, by the approximation properties of the interpolant,

$$
\begin{aligned}
|\tilde{E}(F(x \psi-\eta))| & \leqslant C h^{j+1}\|F\|_{W_{\infty}^{j . h}} \sum_{i=1}^{N} h_{i}\left\|(x \psi)^{\prime}\right\|_{L_{\infty}\left(I_{i}\right)} \\
& \leqslant C h^{j+1}\|F\|_{W_{\infty}^{j . h}}\left(\left\|x \psi^{\prime}\right\|_{L_{1}}+\|\psi\|_{L_{1}}\right) .
\end{aligned}
$$

Altogether the above estimates prove (6.4) and thereby the theorem.

Note that with $k=2 r-3$ in the local quadrature scheme, the $O\left(h^{r}\right)$ convergence of Theorem 4 is preserved in the computational scheme (6.2), and if $k>2 r-3$, the quadrature error is at most $O\left(h^{r+1}\right)$ and thus dominated by the error in the exact solution of (6.1). 
7. Numerical Results. Several test problems have been run using our above methods with piecewise linear approximating functions on uniform partitions. These all show the same qualitative and quantitative behavior.

For the stationary problem, with $b=2,\left\{\varphi_{i}\right\}_{0}^{N-1}$ the basis of $S_{h}$ defined by $\varphi_{i}\left(x_{j}\right)=\delta_{i j}$, and $U_{i}=u_{h}\left(x_{i}\right)$, the symmetric discrete variational problem (2.2) reduces to the difference scheme

$$
\begin{gathered}
\frac{h}{3}\left(U_{0}-U_{1}\right)+\left(x^{2} q \varphi_{0}, \varphi_{0}\right) U_{0}+\left(x^{2} q \varphi_{1}, \varphi_{0}\right) U_{1}=\left(x^{2} f, \varphi_{0}\right), \\
-\frac{1}{h}\left(\bar{x}_{i+1 / 2}^{2}\left(\frac{U_{i+1}-U_{i}}{h}\right)-\bar{x}_{i-1 / 2}^{2}\left(\frac{U_{i}-U_{i-1}}{h}\right)\right) \\
+\frac{1}{h} \sum_{k=i-1}^{i+1}\left(x^{2} q \varphi_{k}, \varphi_{i}\right) U_{k}=\frac{1}{h}\left(x^{2} f, \varphi_{i}\right) \text { for } i=1, \ldots, N-1, \\
U_{N}=0,
\end{gathered}
$$

where $\bar{x}_{i+1 / 2}^{2}=\frac{1}{12}\left(5 x_{i}^{2}+2 x_{i} x_{i+1}+5 x_{i+1}^{2}\right)$. Similarly, the nonsymmetric method takes the form

$$
\begin{gathered}
U_{0}-U_{1}+\left(x q \varphi_{0}, \varphi_{0}\right) U_{0}+\left(x q \varphi_{1}, \varphi_{0}\right) U_{1}=\left(x f, \varphi_{0}\right), \\
-x_{i} \frac{U_{i+1}-2 U_{i}+U_{i-1}}{h^{2}}-2 \frac{U_{i+1}-U_{i-1}}{2 h}+\frac{1}{h} \sum_{k=i-1}^{i+1}\left(x q \varphi_{k}, \varphi_{i}\right) U_{k} \\
=\frac{1}{h}\left(x f, \varphi_{i}\right), \quad i=1, \ldots, N-1, \\
U_{N}=0,
\end{gathered}
$$

and, for instance, using Simpson's rule for the local quadratures with $x_{i+1 / 2} \equiv$ $\left(x_{i}+x_{i+1}\right) / 2, q_{i+1 / 2} \equiv q\left(x_{i+1 / 2}\right)$, and $f_{i+1 / 2} \equiv f\left(x_{i+1 / 2}\right)$,

$$
\begin{gathered}
U_{0}-U_{1}+\frac{h}{3} x_{1 / 2} q_{1 / 2} \frac{U_{0}+U_{1}}{2}=\frac{h}{3} x_{1 / 2} f_{1 / 2}, \\
-x_{i} \frac{U_{i+1}-2 U_{i}+U_{i-1}}{h^{2}}-2 \frac{U_{i+1}-U_{i-1}}{2 h} \\
+\frac{1}{6}\left(x_{i-1 / 2} q_{i-1 / 2} U_{i-1}+\left(x_{i-1 / 2} q_{i-1 / 2}+2 x_{i} q_{i}+x_{i+1 / 2} q_{i+1 / 2}\right) U_{i}\right. \\
\left.\quad+x_{i+1 / 2} q_{i+1 / 2} U_{i+1}\right) \\
=\frac{1}{3}\left(x_{i-1 / 2} f_{i-1 / 2}+x_{i} f_{i}+x_{i+1 / 2} f_{i+1 / 2}\right) \quad \text { for } i=1, \ldots, N-1, \\
U_{N}=0 .
\end{gathered}
$$

In our first example we present some numbers relating to the problem

$$
\begin{gathered}
-u^{\prime \prime}-\frac{2}{x} u^{\prime}+4 u=-4 \text { in } I, \\
u^{\prime}(0)=u(1)=0,
\end{gathered}
$$

the exact solution of which is $u=\sinh 2 x /(x \sinh 2)-1$. The tables below show the distributions of the errors over the interval for our two methods, using $N=10$ and Simpson's rule (which is in fact exact for the nonsymmetric method in this case). 
The symmetric method

\begin{tabular}{|c|c|}
\hline$x$ & $|e(x)|$ \\
\hline 0,0 & 0,004978 \\
0,1 & 0,003170 \\
0,2 & 0,002414 \\
0,3 & 0,001958 \\
0,4 & 0,001628 \\
0,5 & 0,001358 \\
0,6 & 0,001114 \\
0,7 & 0,000873 \\
0,8 & 0,000618 \\
0,9 & 0,000333 \\
\hline
\end{tabular}

Nonsymmetric method

\begin{tabular}{|c|c|}
\hline$x$ & $|e(x)|$ \\
\hline 0,0 & 0,000375 \\
0,1 & 0,000373 \\
0,2 & 0,000368 \\
0,3 & 0,000359 \\
0,4 & 0,000344 \\
0,5 & 0,000323 \\
0,6 & 0,000292 \\
0,7 & 0,000249 \\
0,8 & 0,000190 \\
0,9 & 0,000109 \\
\hline
\end{tabular}

Our next table concerns

$$
-u^{\prime \prime}-\frac{2}{x} u^{\prime}+\left(1-x^{2}\right) u=7-2 x^{2}+x^{4}, \quad u^{\prime}(0)=u(1)=0
$$

with the exact solution $u=1-x^{2}$. It shows maximum mesh points errors for some different values of $N$ and using the trapezoidal rule, the midpoint rule, and Simpson's rule for the quadrature. As predicted by the theory all these schemes preserve the $O\left(h^{2}\right)$ rate of convergence of the exact solution. The results indicate that since the errors in the nonsymmetric method are quite small, it is worthwhile to use the more accurate Simpson's rule.

Symmetric

\begin{tabular}{|l|c|c|c|c|}
\hline & trapez. & midpoint & Simpson & exact \\
\hline$N=5$ & 0,078773 & 0,098332 & 0,039826 & 0,039821 \\
$N=10$ & 0,024214 & 0,030415 & 0,012256 & 0,012257 \\
$N=20$ & 0,007193 & 0,009048 & 0,003635 & 0,003637 \\
$N=40$ & 0,002064 & 0,002577 & 0,001023 & 0,001022 \\
\hline
\end{tabular}

Nonsymmetric

\begin{tabular}{|l|c|c|c|c|}
\hline & trapez. & midpoint & Simpson & exact \\
\hline$N=5$ & 0,040000 & 0,020919 & 0,000729 & 0,000721 \\
$N=10$ & 0,009997 & 0,005257 & 0,000178 & 0,000181 \\
$N=20$ & 0,002495 & 0,001324 & 0,000047 & 0,000053 \\
$N=40$ & 0,000621 & 0,000301 & 0,000009 & 0,000009 \\
\hline
\end{tabular}


Finally, our above semidiscrete methods (using Simpson's rule for the quadrature) were applied to the time-dependent problem

$$
\begin{gathered}
u_{t}-u^{\prime \prime}-\frac{2}{x} u^{\prime}+3 u=\frac{\sinh 2 x}{x \sinh 2}-4 e^{t}+3, \quad x \in I, t>0, \\
u^{\prime}(0, t)=u(1, t)=u(x, 0)=0, \quad t>0,
\end{gathered}
$$

with exact solution $u=\left(e^{t}-1\right) \cdot \sinh 2 x /(x \sinh 2)-e^{t}+1$. For the discretization in time the Crank-Nicolson scheme was used with $\Delta t=1 / 500$.

The first tables show the distribution of the errors over the interval for the two methods at $t=1$ with $N=10$.

Symmetric

Nonsymmetric

\begin{tabular}{|l|c|c|c|}
\hline$x$ & $|e(x)|$ & $x$ & $|e(x)|$ \\
\hline 0,0 & 0,008470 & 0,0 & 0,000713 \\
0,1 & 0,005368 & 0,1 & 0,000707 \\
0,2 & 0,004080 & 0,2 & 0,000699 \\
0,3 & 0,003311 & 0,3 & 0,000682 \\
0,4 & 0,002759 & 0,4 & 0,000656 \\
0,5 & 0,002311 & 0,5 & 0,000618 \\
0,6 & 0,001905 & 0,6 & 0,000560 \\
0,7 & 0,001503 & 0,7 & 0,000479 \\
0,8 & 0,001071 & 0,8 & 0,000366 \\
0,9 & 0,000581 & 0,9 & 0,000211 \\
\hline
\end{tabular}

Our final table shows the maximum mesh point errors at $t=1$ for various choices of $N$.

\begin{tabular}{|l|c|c|}
\hline & Symmetric & Nonsymmetric \\
\hline$N=5$ & 0,027922 & 0,002902 \\
$N=10$ & 0,008469 & 0,000713 \\
$N=20$ & 0,002486 & 0,000177 \\
$N=40$ & 0,000713 & 0,000044 \\
\hline
\end{tabular}

Department of Mathematics

Chalmers University of Technology and

The University of Göteborg

S-412 96 Göteborg, Sweden

1. D. C. Brabston \& H. B. Keller, "A numerical method for singular two-point boundary value problems," SIAM J. Numer. Anal., v. 14, 1977, pp. 779-791.

2. F. R. DE HOOG \& R. WEISs, "On the boundary value problem for systems of ordinary differential equations with a singularity of the second kind," SIAM J. Numer. Anal., v. 11, 1980, pp. 41-60.

3. S. G. Eisenstat, R. S. Schreiber \& M. H. Schultz, Finite Element Methods for Spherically Symmetric Elliptic Equations, Yale Computer Science Report No. 109, 1977.

4. B. Gustafsson, "A numerical method for solving singular boundary value problems," Numer. Math., v. 21, 1973, pp. 328-344. 
5. P. JAMET, "On the convergence of finite-difference approximations to one-dimensional singular boundary-value problems," Numer. Math., v. 14, 1970, pp. 355-378.

6. D. JESPERSEN, "Ritz-Galerkin methods for singular boundary value problems," SIAM J. Numer. Anal., v. 15, 1978, pp. 813-834.

7. R. D. Russel \& L. F. Shampine, "Numerical methods for singular boundary value problems," SIAM J. Numer. Anal., v. 12, 1975, pp. 13-36.

8. R. SChreIBER \& S. C. EISENSTAT, "Finite element methods for spherically symmetric elliptic equations," SIAM J. Numer. Anal., v. 18, 1981, pp. 546-558.

9. M. F. WHEELER, “ $L_{\infty}$ estimates of optimal orders for Galerkin methods for one-dimensional second order parabolic and hyperbolic equations," SIAM J. Numer. Anal., v. 10, 1973, pp. 908-913. 